\title{
38. Partial Alteration of a Protein Streptomyces Subtilisin Inhibitor by Site-Directed Mutagenesis
}

\author{
By Kin-ichiro Miura, Izumi KumagaI, Shusei Obata, \\ Shuichi KoJima, and Seiichi Taguchi \\ Department of Industrial Chemistry, Faculty of Engineering, \\ The University of Tokyo, Hongo, Tokyo 113 \\ (Communicated by Motoo Kimura, M. J. A., June 14, 1988)
}

A protease inhibitor produced by Streptomyces albogriseolus was isolated by Murao and Sato ${ }^{1)}$ in 1971 . It is a protein consisted of 110 amino acids. Since it inhibits a protease subtilisin specifically, it is called as Streptomyces subtilisin inhibitor, SSI. Its tertiary structure as well as primary structure has been studied well by means of various physical techniques, which includes X-ray analysis on its crystal. Documents of the research on SSI carried out by ccoperation of Japanese investigators were well summarized in a book, "Protein Protease Inhibitor-The Case of Streptomyces Subtilisin Inhibitor (SSI)",2) issued by Publisher Elsevier in 1985. Standing on these precise studies on the structure and the function, we selected SSI as a target for protein engineering.

Cloning of SSI gene. First of all we started from cloning of the SSI gene. DNA was isolated from S. albogriseolus by Saito-Miura's method which used pH 9-phenol for efficient extraction of DNA and ribonucleases for complete elimination of RNA. ${ }^{3)}$ Streptomyces DNA was digested with restriction endonucleases SalI and Bgl II. DNA fragments were combined with E. coli plasmid pBR322, which was treated with the restriction endonucleases, SalI and BamHI, and linked with DNA repairing enzymes. The recombinant plasmids were inccrporated into $E$. coli. SSI gene was searched by annealing with the probes, 26 and 29 base long, respectively, which were synthesized chemically, incorporating inosinic acid into third letter position of a codon to read degenerated bases. pSI30 containing SSI gene was cloned. The SSI gene was once more cloned by the plasmid pUC18. Thus, pSI51 and pSI52 with opposite orientation of SSI gene were obtained.

Structure of SSI gene. Nucleotide sequence of the SSI gene was analysed mainly by the "dideoxy" method. The corresponding amino acid sequence was the same to the sequence obtained by Ikenaka et al. ${ }^{4)}$ with the analysis of amino acids (Fig. 1). The initiation codon ATG precedes 31 amino acid codons upstream of $\mathrm{N}$ terminal of SSI. These 31 amino acids would correspond to the signal peptide. In the used clone, there were 583 bases upstream of SSI gene, which were considered as sufficient length for expression of the gene. The base sequence of SSI gene was quite rich in GC content (73\%) as whole DNA of Streptomyces. The GC content of third letter in codons reached $96 \%$.

Expression of SSI gene. The cloned SSI gene was incorporated in a Streptomyces multicopy plasmid (pIJ702) and transferred into Streptomyces lividans. SSI was well expressed in this system and secreted outside of the bacterium cell. It was purified as a single protein, which reacted with the antibody produced by SSI. The size of this SSI expressed in S. lividans was 3 amino acids long to upstream of the $\mathrm{N}$-terminal amino acid of the ordinary SSI, which was 


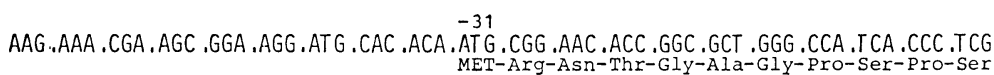
-20 TT Val-Ser-Arg-Pro-Pro-Pro-Ser-Ala-Ala-Pro-Leu-Ser-Gly-Ala-Ala-Leu-Ala-Ala-Pro-Gly 1101020

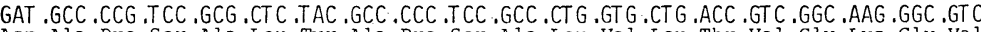
Asp-Ala-Pro-Ser-Ala-Leu-Tyr-Ala-Pro-Ser-Ala-Leu-Val-Leu-Thr-Val-Gly-Lys-Gly-Val

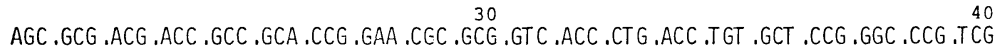
Ser-Ala-Thr-Thr-Ala-Ala-Pro-Glu-Arg-Ala-Val-Thr-Leu-Thr-Cys-Ala-Pro-Gly-Pro-Ser GGC .ACC .CAC .CCG .GCG .GCC ,GGC .TCG .GCC .TGC .GCG .GAC .CTG .GCC .GCC .GTC .GGC .GGC .GAC .CTG Gly-Thr-His-Pro-Ala-Ala-Gly-Ser-Ala-Cys-Ala-Asp-Leu-Ala-Ala-Val-Gly-Gly-Asp-Leu

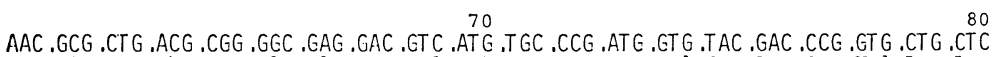
Asn-Ala-Leu-Thr-Arg-Gly-Glu-Asp-Val-Met-Cys-Pro-Met-Val-Tyr-Asp-Pro-Val-Leu-Leu

90

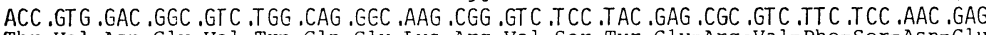
Thr-Val-Asp-Gly-Val-Trp-Gln-Gly-Lys-Arg-Val-Ser-Tyr-Gly-Arg-Val-Phe-Ser-Asn-Glu TGC ,GAG .ATG .AAC ,GCG .CAC .GGC .TCG .AGC .GTC .TTC .GCC .TTC .TAG . Cys-Glu-Met-Asn-Ala-His-Gly-Ser-Ser-Val-Phe-Ala-Phe-xxx-

Fig. 1. Primary structure of SSI and its gene.

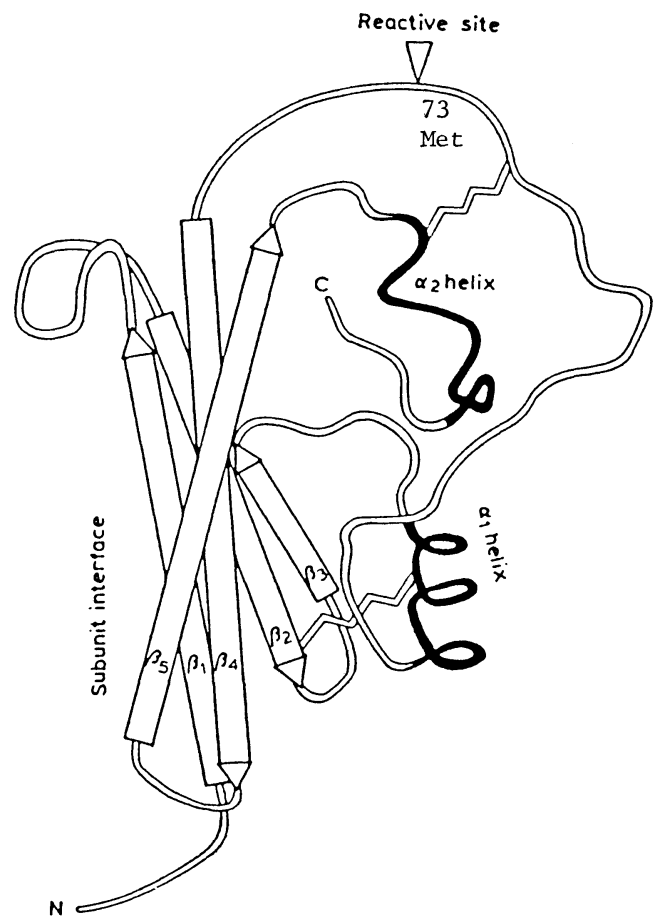

Fig. 2. A schematic drawing of SSI by T. Komiyama. ${ }^{2)}$ 

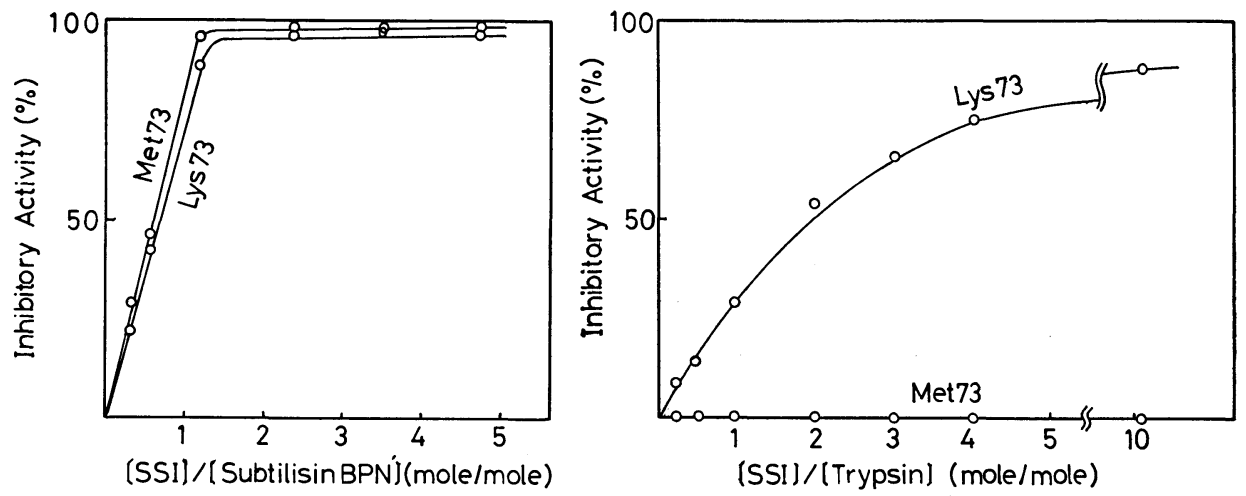

Fig. 3. Inhibition of subtilisin (left) and trypsin (right) by wild type SSI (Met73) and mutant SSI (Lys73).

expressed in $S$. albogriseolus.

Replacement of an amino acid by gene engineering. 73 Met in SSI is known as the reaction site to subtilisin (Fig. 2). The nucleotide sequence of ATG corresponding to Met at the position 73 was converted into AAG corresponding to Lys by gene engineering. The altered gene was also expressed well in $S$. lividans. Inhibition for subtilisin of the 73 Lys-SSI was almost the same to the wild type SSI. Although the wild type of SSI does not inhibit trypsin, the mutant 73 Lys-SSI inhibits trypsin (Fig. 3). Ki value for trypsin was $1 \times 10^{-4}$ with 73 Met-SSI, but $1 \times 10^{-8}$ to $4 \times 10^{-9}$ with 73 Lys-SSI. Similar change was also observed for lysyl endopeptidase. The mutant SSI substituted 73 Met to Arg inhibited trypsin as well as subtilisin, but not lysyl endopeptidase.

Functional specificity of SSI was changed by substitution of one amino acid in the reaction site for a protease.

The authors thank Dr. Shin-ichi Ishii, Prof. Keitaro Hiromi, Dr. Yukio Mitsui and Dr. Masatsune Kainosho for their valuable advices.

\section{References}

1) Murao, S., and Sato, S. (1973): Agr. Biol. Chem., 37, 1067.

2) Hiromi, K. et al. (eds.) (1985) : Protein Protease Inhibitor-The Case of Streptomyces Subtilisin Inhibitor (SSI). Elsevier Science Publishers, Amsterdam.

3) Saito, H., and Miura, K. (1963) : Biochim. Biophys. Acta, 72, 619.

4) Ikenaka, T. et al. (1974): J. Biochem., 76, 1191. 